\title{
DISFAGIA NA DOENÇA DE CHARCOT-MARIE-TOOTH: RELATO DE CASO
}

\section{DYSPHAGIA IN CHARCOT-MARIE-TOOTH DISEASE: A CASE REPORT}

\author{
Karoline Terezinha Quaresma ${ }^{1}$, Júlia Fabre Renke ${ }^{1}$, \\ Simone Augusta Finard ${ }^{1}$
}

\section{RESUMO}

A doença de Charcot-Marie-Tooth (CMT) é uma polineuropatia motora, sensitiva, hereditária e de predomínio distal. Os membros inferiores são os mais afetados e, ocasionalmente, há comprometimento dos membros superiores. Este estudo descreve a presença de disfagia orofaríngea e a intervenção fonoaudiológica em uma paciente de 58 anos com diagnóstico de CMT, encaminhada à avaliação fonoaudiológica devido a queixas na deglutição. Na avaliação inicial identificaram-se alterações, tanto de caráter estrutural quanto funcional, que resultaram em uma disfagia orofaríngea leve. Também se utilizou o Questionário de Qualidade de Vida na Disfagia para identificar o impacto na qualidade de vida da paciente. Após um mês de acompanhamento observou-se melhora dos sintomas e aumento do conforto e segurança ao deglutir.

Palavras-chave: Transtornos de deglutição; Doença de Charcot-Marie-Tooth; Fonoaudiologia

\begin{abstract}
Charcot-Marie-Tooth (CMT) disease is a motor and sensory, hereditary, distally predominant polyneuropathy. The lower limbs are most affected and, occasionally, there is upper limb impairment; however, presence of oropharyngeal dysphagia has been identified. The present study describes the findings and speech therapy intervention in a 58-year-old patient, with CMT diagnosis, referred to speech therapy evaluation due to swallowing complaints. In an initial evaluation, presence of structural and functional alterations, resulting in mild oropharyngeal dysphagia, was identified. The Swallowing Quality of Life questionnaire was also used to identify the impact of dysphagia in the patient's quality of life. After a month of follow-up, an improvement was observed in the presenting symptoms, as well as an increase in comfort and safety during swallowing.
\end{abstract}

Keywords: Deglutition disorders; Charcot-Marie-Tooth disease; Speech, language and hearing sciences

\section{INTRODUÇÃO}

A doença de Charcot-Marie-Tooth (CMT) é uma polineuropatia motora e sensitiva hereditária de predomínio distal, com prevalência estimada de 40/100,000 pessoas ${ }^{1}$. De acordo com a natureza da lesão do nervo periférico, a CMT é classificada em tipo 1, se desmielinizante, e tipo 2, se com presença de degeneração axonal ${ }^{2}$. A classificação também é realizada identificando-se o padrão de transmissão e a base genética. Além da herança autossômica dominante ou recessiva, há casos da doença ligada ao cromossomo $X$ $(\mathrm{CMTX})^{3}$. O quadro típico da doença é caracterizado por atrofia muscular distal progressiva, fraqueza, arreflexia e alterações sensitivas de graus variados. Os membros inferiores são mais afetados e, ocasionalmente, há comprometimento dos membros superiores.

Clin Biomed Res. 2021;41(2):181-184 1 Serviço de Fisiatria e Reabilitação, Hospital de Clínicas de Porto Alegre. Porto Alegre, RS, Brasil.

Autor correspondente: Simone Augusta Finard sfinard@hcpa.edu.br Serviço de Fisiatria e Reabilitação Hospital de Clínicas de Porto Alegre Rua Ramiro Barcelos, 2350 90035-903, Porto Alegre, RS, Brasil. 
Em pacientes com CMTX, cerca de $11 \%$ podem apresentar acometimento do Sistema Nervoso Central (SNC), por mutação da proteína $\mathrm{Cx32}$ relacionada a integridade e funcionalidade da mielina tanto de nervos periféricos quanto centrais ${ }^{4}$. As manifestações neurológicas mais comuns encontradas nesses casos foram transientes e incluíram disfagia, disartria, ataxia, hemiparesia e tetraparesia ${ }^{4}$.

O tratamento é baseado na reabilitação e prevenção, primordialmente, de deformidades dos membros inferiores e superiores ${ }^{5}$. Quanto à intervenção fonoaudiológica, são escassos os estudos, sendo preconizado o uso de técnicas recomendadas na reabilitação da deglutição em pacientes com doenças neurológicas.

O objetivo deste trabalho é descrever os achados e a intervenção fonoaudiológica em um caso de CMT com presença de disfagia. Cabe ressaltar que, embora apareça de forma transitória, trata-se de um sintoma que impacta na qualidade de vida e, dependendo do grau de comprometimento, pode desencadear quadros de desnutrição, desidratação ou pneumonia ${ }^{4,6}$.

\section{RELATO DO CASO}

Paciente de 58 anos de idade, do sexo feminino, com diagnóstico de CMT pela equipe de Neurologia do hospital de referência desde os 38 anos. Foi encaminhada à avaliação fonoaudiológica devido queixas na deglutição. Em entrevista inicial, referiu histórico de bruxismo, com necessidade de uso de placa dentária noturna, e tensão da musculatura cervical.

À oroscopia, apresentou oclusão dentária alterada, uso de prótese dentária superior parcial e ausência de molares e pré-molares inferiores. Na avaliação miofuncional e estrutural, identificou-se hipotonia da língua e presença de tremor ao movimento; suporte respiratório reduzido e qualidade vocal rugosa e áspera.

$\mathrm{Na}$ avaliação da deglutição de saliva, observou-se excursão laríngea reduzida com a técnica de "quatro dedos", além de atraso do disparo ao deglutir e ausculta cervical com sinais de esforço. Dentre os alimentos testados nas consistências líquida, pastosa e sólida, constataram-se alterações apenas durante o consumo da consistência sólida. Observou-se fases preparatória oral e oral lentificadas, seguidas de deglutições múltiplas com tendência à extensão cervical e excursão laríngea reduzida, além de ausculta cervical alterada por sinais de esforço ao deglutir.

Após a finalização das avaliações, o diagnóstico fonoaudiológico foi de disfagia orofaríngea leve, sendo mantida a alimentação por via oral total. Utilizou-se também o Questionário de Qualidade de Vida na Disfagia, SWAL-QOL ${ }^{7}$, constituído de 44 questões divididas em 11 domínios, para caracterizar o impacto do distúrbio da deglutição. Foram identificadas alterações referentes a sete domínios (Tabela 1).
Tabela 1: Itens do Swal-Qol com alteração encontrados na aplicação pré e pós fonoterapia.

\begin{tabular}{|c|c|c|c|}
\hline $\begin{array}{l}\text { Domínio } \\
\text { Avaliado }\end{array}$ & Questões & $\begin{array}{c}\text { Resposta } \\
\text { pré } \\
\text { fonoterapia* }\end{array}$ & $\begin{array}{c}\text { Resposta } \\
\text { pós } \\
\text { fonoterapia* }\end{array}$ \\
\hline \multirow[t]{2}{*}{$\begin{array}{l}\text { Alimentação } \\
\text { como um } \\
\text { fardo }\end{array}$} & $\begin{array}{l}\text { Lidar com } \\
\text { o meu } \\
\text { problema de } \\
\text { deglutição é } \\
\text { muito difícil. }\end{array}$ & 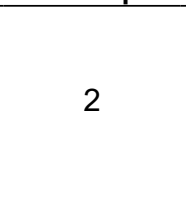 & 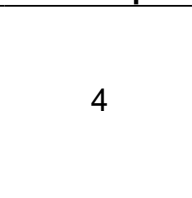 \\
\hline & $\begin{array}{l}\text { Meu } \\
\text { problema de } \\
\text { deglutição } \\
\text { é a maior } \\
\text { perturbação } \\
\text { de minha } \\
\text { vida. }\end{array}$ & 3 & 4 \\
\hline \multirow[t]{3}{*}{$\begin{array}{l}\text { Frequência } \\
\text { dos } \\
\text { sintomas }\end{array}$} & $\begin{array}{l}\text { Engasgo } \\
\text { quando me } \\
\text { alimento. }\end{array}$ & 3 & 4 \\
\hline & $\begin{array}{l}\text { A comida } \\
\text { para na } \\
\text { garganta. }\end{array}$ & 1 & 3 \\
\hline & $\begin{array}{l}\text { As pessoas } \\
\text { têm }\end{array}$ & & \\
\hline Comunicação & $\begin{array}{l}\text { dificuldade } \\
\text { em me } \\
\text { entender. }\end{array}$ & 2 & 3 \\
\hline $\begin{array}{l}\text { Medo de } \\
\text { alimentar-se }\end{array}$ & $\begin{array}{l}\text { Tenho medo } \\
\text { de engasgar } \\
\text { quando me } \\
\text { alimento. }\end{array}$ & 2 & 5 \\
\hline \multirow[t]{3}{*}{$\begin{array}{l}\text { Saúde } \\
\text { mental }\end{array}$} & $\begin{array}{l}\text { Meu } \\
\text { problema de } \\
\text { deglutição } \\
\text { me deprime. }\end{array}$ & 2 & 4 \\
\hline & $\begin{array}{l}\text { Ter que tomar } \\
\text { muito cuidado } \\
\text { quando bebo } \\
\text { ou como me } \\
\text { aborrece. }\end{array}$ & 2 & 4 \\
\hline & $\begin{array}{l}\text { Tenho estado } \\
\text { desanimado } \\
\text { com meu } \\
\text { problema de } \\
\text { deglutição. }\end{array}$ & 3 & 5 \\
\hline Sono & $\begin{array}{l}\text { Tem } \\
\text { problemas } \\
\text { para dormir? }\end{array}$ & 3 & 5 \\
\hline \multirow[t]{2}{*}{ Fadiga } & $\begin{array}{l}\text { Sente-se } \\
\text { exausto? }\end{array}$ & 2 & 5 \\
\hline & $\begin{array}{l}\text { Sente-se } \\
\text { fraco? }\end{array}$ & 2 & 1 \\
\hline- & $\begin{array}{l}\text { Você diria } \\
\text { que a sua } \\
\text { saúde é: }\end{array}$ & $1^{* *}$ & $3^{* * *}$ \\
\hline
\end{tabular}

${ }^{*}$ Respostas de acordo com escala de frequência do protocolo (sempre [1], muitas vezes [2], algumas vezes [3], um pouco [4], nunca [5]).

${ }^{* *}$ Ruim.

${ }^{* * *}$ Boa. 
Do ponto de vista terapêutico, iniciou-se com orientações quanto aos cuidados para deglutições seguras com captação de porções pequenas, posicionamento adequado durante a refeição e ajustes para o preparo do bolo alimentar. Prescreveuse exercício isotônico para aumento da amplitude da excursão laríngea, uso da manobra postural de flexão cervical durante a deglutição de alimentos sólidos e de manobra de limpeza com deglutições múltiplas e com esforço, para prevenir estase em recessos faringolaríngeos.

Durante um mês de acompanhamento, com um atendimento semanal de 40 minutos, foi realizado treino para deglutições seguras com uso dos alimentos habituais de consumo da paciente, revisão da execução da prescrição e reforço das recomendações a serem realizadas durante a refeição. Como resultado desta abordagem, observou-se que, à ausculta cervical durante a deglutição, ainda havia sinais de esforço, mas sem queixas por parte da paciente, além da diminuição do tempo na fase preparatória do alimento.

Nas respostas da reaplicação do SWAL-QOL, identificou-se que a paciente apresentava maior conforto e segurança ao deglutir e melhor classificação do seu estado de saúde global. Observou-se piora apenas no item relacionado à percepção sobre a fraqueza. Os resultados obtidos na aplicação pré e pós-fonoterapia estão detalhados na Tabela 1.

\section{DISCUSSÃO}

Os resultados da avaliação clínica estrutural e miofuncional estão em concordância com os obtidos no estudo em que cerca de $40 \%$ dos pacientes apresentaram paralisia de pregas vocais, disfonia, além de fraqueza nos músculos mastigatórios e faciais ${ }^{8}$.

Em relação às alterações vocais, fez-se a hipótese de Disfonia por Tensão Muscular, uma vez que a paciente referia diagnóstico de bruxismo e tensão muscular. A tensão exacerbada das zonas perilaríngea e cervical ocasionaria um aumento da rigidez muscular $^{9}$, e as características perceptivoauditivas da voz seriam rouquidão, soprosidade, instabilidade e aspereza ${ }^{10}$, dois deles apresentados pela paciente.

$\mathrm{Na}$ avaliação funcional da deglutição, encontraramse alterações nas fases preparatória oral, oral e faríngea. A ausência de pré-molares e molares pode justificar a fase preparatória oral mais lentificada, assim como a presença de hipotonia dos músculos mastigatórios, já encontrado em outros casos de $\mathrm{CMT}^{8}$. Há relatos também, apesar de infrequentes, do acometimento de nervos cranianos, o que explicaria não só as alterações da fase preparatória oral como também da fase oral e faríngea encontradas ${ }^{11,12}$.

$\mathrm{Na}$ aplicação do questionário SWAL-QOL préfonoterapia, foram encontradas alterações nas questões para grande parte dos domínios avaliados. Em estudos que relacionaram a qualidade de vida com as alterações físicas na CMT, os escores de qualidade de vida foram significativamente menores que na população saudável ${ }^{13}$. Já em estudo comparativo ${ }^{14}$, houve maior impacto na qualidade de vida nas esferas social e emocional, em concordância com os domínios comunicação e saúde mental alterados no SWAL-QOL deste caso.

Acerca da melhora na deglutição pós-fonoterapia, esta pode também estar associada ao caráter transitório das manifestações de origem neurológica na doença de CMT, as quais podem ter duração desde horas a semanas ${ }^{4}$.

Neste contexto, ressalta-se a importância de identificar as alterações da deglutição na CMT, mesmo que a disfagia seja um sintoma transiente, encontrado em manifestações menos prevalentes desta doença, a fim de manter a segurança e a funcionalidade da deglutição e, por consequência, qualidade de vida com prazer no consumo alimentar.

\section{REFERÊNCIAS}

1. Martyn CN, Hughes RA. Epidemiology of peripheral neuropathy. J Neurol Neurosurg Psychiatry. 1997;62(4):310-8.

2. Harding AE, Thomas PK. The clinical features of hereditary motor and sensory neuropathy types I and II. Brain. 1980;103(2):259-80.

3. Vallat JM, Funalot B. Charcot-MarieTooth (CMT) disease: an update. Med Sci (Paris). 2010;26(10):842-7.

4. Al-Mateen M, Craig AK, Chance PF. The central nervous system phenotype of X-linked Charcot-Marie-
Tooth disease: a transient disorder of children and young adults. J Child Neurol. 2014;29(3):342-8.

5. Ouvrier R, Geevasingha N, Ryan MM. Autosomal-recessive and $\mathrm{X}$-linked forms of hereditary motor and sensory neuropathy in childhood. Muscle Nerve. 2007;36(2):131-43.

6. Silva BF, Finard SA, Olchik MR Qualidade de vida em pacientes com doença de Machado-Joseph sob acompanhamento fonoaudiológico para disfagia. Rev Cefac. 2016;18(4):992-1000.
7. Portas JG. Validação para a língua portuguesa brasileira dos questionários: qualidade de vida em disfagia (Swal-qol) e satisfação do paciente e qualidade do cuidado no tratamento da disfagia (Swal-care) [Dissertação]. São Paulo: Fundação Antônio Prudente; 2009.

8. Pareyson D, Stojkovic T, Reilly MM, Leonard-Louis S, Laura M, Blake J, et al. A multicenter retrospective study of charcot-marie-tooth disease type 4B (CMT4B) associated with mutations in myotubularin-related proteins (MTMRs). Ann Neurol. 2019;86(1):55-67. 
9. Hirano M. Clinical examination of voice. New York: Springer Verlag; 1981.

10. Behlau M, Pontes P. Avaliação e tratamento das disfonias. São Paulo: Lovise; 1995.

11. Das N, Kandalaft S, Wu X, Malhotra A. Cranial nerve involvement in CharcotMarie-Tooth Disease. J Clin Neurosci. 2017;37:59-62.
12. Benson B, Sulica L, Guss J, Blitzer A. Laryngeal neuropathy of CharcotMarie-Tooth disease: further observations and novel mutations associated with vocal fold paresis. Laryngoscope. 2010;120(2):291-6.

13. McCorquodale D, Pucillo EM, Johnson NE. Management of Charcot-MarieTooth disease: improving long-term care with a multidisciplinary approach. J Multidiscip Healthc. 2016;9:7-19.

14. Taniguchi JB, Elui VMC, Osorio FL, Hallak JEC, Crippa JAS, Machadode-Souza JP, et al. Quality of life in patients with Charcot-Marie-Tooth disease type 1A. Arq Neuropsiquiatr. 2013;71(6):392-6.

Recebido: 25 set, 2020

Aceito: 22 fev, 2021 